\title{
Research on the development mode of digital channels of commercial banks based on blockchain finance
}

\author{
Shuaiyin Yang ${ }^{1, a}$, Xiaoyang Han ${ }^{2, \text { b* }}$, Gengyuan Tian ${ }^{3, c}$, Haiyue Tuo ${ }^{4, d}$ \\ ${ }^{1}$ School of Economics and Trade, Henan University of Technology, Zhengzhou, China \\ ${ }^{2}$ School of Economics and Trade, Henan University of Technology, Zhengzhou, China \\ ${ }^{3}$ School of Economics and Trade, Henan University of Technology, Zhengzhou, China, \\ ${ }^{4}$ School of Economics and Trade, Henan University of Technology, Zhengzhou, China
}

\begin{abstract}
In 2021, blockchain was included in the national five-year plan for the first time, and in the chapter of "Accelerating Digital Development and Building Digital China", blockchain was listed as one of the seven key industries of digital economy in the 14th Five-Year Plan. The rapid development of blockchain technology not only promotes the high-speed operation of China's real economy and greatly improves the overall service quality of China's digital economy, but also provides new development ideas for the innovative development of digital channels of commercial banks. In this paper, through the use of literature research method and qualitative analysis method, we deeply analyze the challenges faced by the development mode of commercial banks at the present stage, etc. Accordingly, we propose corresponding countermea-sures and suggestions from the perspectives of research and development of technology, customer privacy protection and improvement of service efficiency, so as to continuously empower the digital transformation of commercial banks in China.
\end{abstract}

\section{INTRODUCTION}

In 2021, blockchain was included in the 14th Five-Year Plan for the first time. To a certain extent, this is not only a confirmation of the future development prospect of blockchain, but also a hint that the world has gradually leaped from the information age to the digital age, and the development of digital economy has become the wave of the times. The digital application represented by blockchain is gradually extended to various industries, among which the joint innovation application between banking industry and blockchain is the most common, and the influence of blockchain finance on the business development of commercial banks in the new era is also increasingly strengthened.

Blockchain, as a kind of chain data structure that connects data blocks in a sequential way in chronological order, is a distributed ledger with the advantages of immutability, fairness and transparency, and privacy protection based on cryptogram-phy as a guarantee [1]. Due to the rapid take-off of information technology, the blockchain industry in the era of digital economy is developing rapidly. According to the relevant data : the number of blockchain-related enterprises in China has reached 113,185 in 2020, and the number is amazing while the industry market scale is also hitting a peak year by year. From the perspective of the overall application direction of the industry, the impact and application of blockchain

aysy13290945661@163.com

${ }^{* b}$ Corresponding author: hxy1342438651@163.com

ca1324766218@163.com

dtuot1216@163.com technology on the banking industry is the most significant. As a model of the banking industry, blockchain finance under the combination of commercial banks and blockchain is bound to have a broader market and future in the future. At present, the main application channels of blockchain finance include: supply chain finance, credit asset business, cross-border payment, etc. For commercial banks, it greatly improves the overall operational efficiency of commercial banks while reducing transaction costs and improving risk pricing level, etc. Therefore, the exploration of digital channel development mode of commercial banks combined with blockchain technology application is essential.

Blockchain finance has been a hot topic year by year in recent years, and the current financial industry scholars focus more on the analysis of the impact of blockchain technology on the financial industry section.

It ignores the analysis of the specific development application of blockchain finance for commercial banks and the study of what digital channel development mode should be adopted for large commercial banks under blockchain technology. This paper takes the development of blockchain technology as the background, and analyzes the necessity of digital channel development of commercial banks, the probl-ems in the development mode and the corresponding develop-ment suggestions, hoping to inspire the later scholars on the development of blockchain technology in commercial banks. 


\section{THE NECESSITY OF CHANGING THE DIGITAL DEVELOPMENT CHANNEL MODEL OF COMMERCIAL BANKS}

Blockchain, as a new technology combination, integrates new technologies such as $\mathrm{P} 2 \mathrm{P}$ network, consensus algorithm, asymmetric encryption, smart contract, etc. It has the character-istics of distributed peer-to-peer, chain data block, anti-forgery and anti-tampering, traceability, transparency and trustworthy-ness and high reliability, etc. Its technical characteristics have great advantageous features in the supply chain finance scenario, and these features have launched great challenges for the existing business of commercial banks Therefore, the transformation and upgrading of the current digital technology development model of commercial banks is necessary [2].

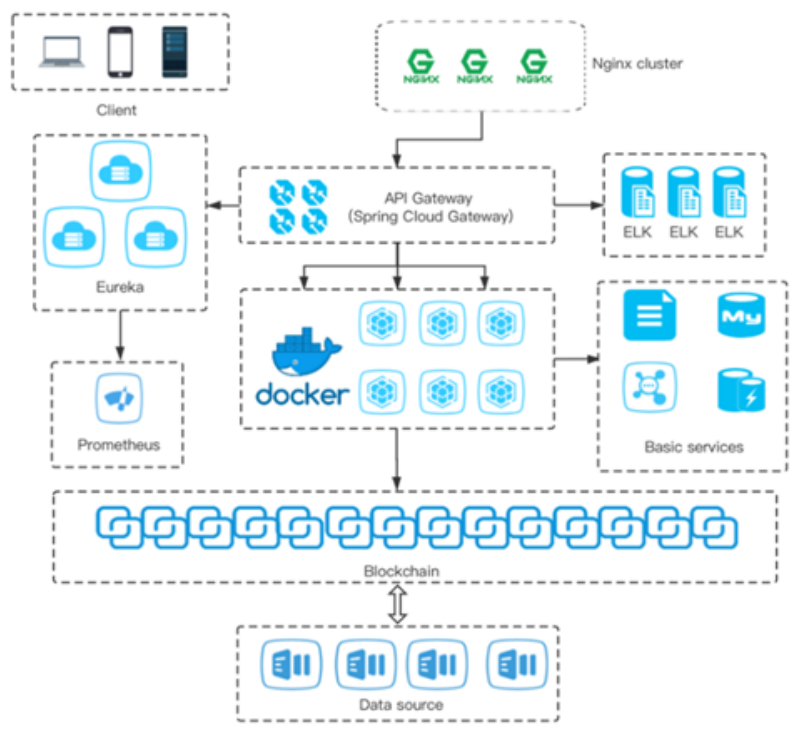

Figure 1. Blockchain technology architecture diagram.

\subsection{The development level of blockchain-based digital business of commercial banks is low}

From the business point of view, most of the current digital businesses of commercial banks are still only shifting part of their offline business to online development and converting manual services into artificial intelligence services, which is far from being able to meet the personalized and customized needs of corporate investment and financing, and cannot provide comprehensive and multi-level professional services for enterprises, which greatly limits the expansion and innovative development of the digital business of commercial banks.

From the historical and functional point of view, the most important function of each commercial bank after its creation is to absorb deposits and lending, to absorb the deposits of depositors, and then to issue loans outward in turn to earn interest rate differential. And in the context of blockchain development, if people are clearing transactions on the blockchain, then the digital currency of these transactions on the blockchain is only equivalent to the numbers in the super ledger, and these numbers are not dependent on the bank accounts of various commercial banks to exist. This also means that the transaction currencies in the blockchain bypass the accounts of traditional commercial banks, and they are independent digital currencies that are independent of the banking system. If these digital currencies in the blockchain can achieve liquidity and stability combined with independence and security, then the blockchain will inevitably affect the traditional function of commercial banks of absorbing residents' savings and issuing loans, leading to a serious impact on the native business of commercial banks.

\subsection{The current technology of commercial banks cannot guarantee the security and stability of the data system}

Big Data has brought people to the era of data collection, processing, analysis and sharing, but at the same time it has also caused serious problems of information and data leakage and abuse. The contradiction between the privacy protection of user information and data and data sharing has become a pressing problem for the society nowadays. At present, the digital technology of commercial banks is still at a relatively preliminary stage, and they are not able to establish a safe and effective user information encryption and privacy protection system, which makes the public have great concerns about the security of commercial bank funds and the privacy of personal information, and limits the further development of digital business of commercial banks.

And the zero-knowledge proof algorithm in encryption technology in the background of blockchain can effectively help the leakage of user information, and its role is to be able to ensure that the user's personal key cannot be cracked, as long as we can ensure that the preliminary parameter information of the system is not leaked, we can ensure the security of user information. And with the continuous improvement and innovation of blockchain technology in the field of security, the protection of customers' private information and the need for secure data sharing will force commercial banks to layout to the digital security field based on blockchain.

\subsection{The digital service eco-chain of commercial banks has not yet formed a perfect closed loop}

Under the impetus of digital economy, creating a customercentered integrated and customized ecosystem will become the main trend for the development of commercial banks in the future. The current service platforms of commercial banks are still at the stage of small service scope, scattered content and misaligned service positioning, failing to form an ecological closed loop with high standard and strict supervision that combines point and line with customer demand, highly focused service content and precise positioning. With the impetus of blockchain technology, commercial banks must create a highly decentralized or completely decentralized system, make full use of the decentralization, smart contract, transparency and trustworthiness of blockchain, take the lead in technology exploration and innovation, seize the first opportunity of 
this technological innovation, and promote the rapid transformation and upgrading of digital business development model [3].

\section{CHALLENGES OF DIGITAL TRANSFORMATION OF COMMERCIAL BANKS BASED ON BLOCKCHAIN FINANCE}

\subsection{Customer privacy leakage problem}

Due to the nature of blockchain technology itself, when customers use blockchain for transaction, every transaction will be presented in the block, and the whole transaction process will be forced to be displayed in the public view, which will inevitably cause the leakage of customers' privacy transactions. Commercial banks inevitably face the confrontation between data security protection by distributed ledger and customer privacy leakage in the transaction. On the one hand, the private key becomes the unique identification of the ownership and dominion of the customer's assets under the blockchain transaction, and the loss or leakage of the private key will cause the loss of the customer's assets, while in the actual transaction, ensuring the security of the private key intact is a headache for enterprises or individuals. In addition, the development of emerging technologies such as quantum computers in the future will make the cracking of asymmetric algorithms in blockchain technology easy, which will cause incalculable leakage losses to customer information and assets. On the other hand, the transparency of blockchain technology and the privacy of customers are opposing dilemmas that are difficult to be resolved. All the transaction information of customers is recorded on the corresponding blockchain public chain. Although commercial banks try to solve the problem by adopting the method of "pass-through", this method of protecting privacy at the expense of blockchain value does not fully utilize the superiority of blockchain technology, therefore, actively coordinating the problem of customer privacy leakage and data sharing will be the future of commercial banks. Therefore, the active coordination of customer privacy and data sharing will be the top priority for commercial banks to develop blockchain finance in the future.

\subsection{Difficulty in matching the demand and supply sides of technology}

As far as the current development stage is concerned, blockchain technology is still in the development and waitand-see stage, and there are many technically unsolvable problems in combining with the development of commercial banks, such as system security issues, network security issues, and block capacity overload issues.

For the system security issue, blockchain technology usually needs to invest a large amount of computer resources to carry out batch processing of information, and the current computer development cannot meet such a high computing demand, and there may be corresponding loopholes in the whole system algorithm, and once the number of nodes involved in the system cannot meet the demand of the system or the corresponding preventive measures of the system are not perfect, these potential If the number of nodes involved in the system does not meet the needs of the system or the corresponding preventive measures of the system are not perfect, these potential problems will be exposed and cause serious security incidents to the system.

For cyberspace security issues, because the development of blockchain technology is not yet mature, there are still many potential network loopholes in the overall mechanism of blockchain technology, which creates conditions for criminals' criminal activities. The anonymity and decentralization trend of blockchain technology create even more problems for regulators in managing constraints. The anonymity of blockchain technology makes it difficult for regulators to track the real identity information of criminals, and the decentralization trend of blockchain technology weakens the role and power of regulators in supervision, leading to frequent cyber security incidents and causing serious cyberspace security problems.

\subsection{The development of blockchain technology lacks corresponding regulatory measures}

The smooth development of digital development channel model of commercial banks cannot be separated from matching laws and regulations and reasonable regulatory mechanism. Along with the strengthening of regulation of blockchain technology by regulators and the gradual improvement of relevant laws and regulations, all of them are bound to be important factors affecting the digital transformation of commercial banks. At present, the development of blockchain finance lacks corresponding regulatory measures, and the reasons for this situation are as follows: on the one hand, the development characteristics of blockchain technology itself are in conflict with the existing regulatory mechanism, and the "decentralization" characteristics of blockchain technology greatly weaken the regulatory function of regulators and the influence of national macro-control [4]. The "decentralized" feature of blockchain technology greatly weakens the regulatory function of regulators and the influence of national macroeconomic regulation, and even impacts the existing monetary policy, which inevitably leads regulators to maintain a cautious attitude when developing blockchain technology. On the other hand, the three major ones on May 19, 2021, further indicate that the attitude of regulatory authorities towards blockchain technology is to strengthen regulation. As the research on blockchain technology is still in the early stage of development and the technology is not yet mature, many countries hold a cautious attitude towards the development, which leads to the lack of supervision of the corresponding laws and regulations in the current development stage of blockchain, which undoubtedly increases the business risks of commercial banks. The statement of regulatory authorities will certainly bring strong control of blockchain technology and bring higher standard of development difficulties for the transformation of digital development mode of commercial banks. 


\section{COUNTERMEASURE SUggestions FOR COMMERCIAL BANKS TO EXPLORE DIGITAL DEVELOPMENT CHANNELS}

The development of blockchain finance plays a crucial role in the development of digital economy for commercial banks, and the wide application of blockchain finance in the banking industry is a general trend. Commercial banks should actively explore digital development channels and actively formulate relevant development strategies in order to maintain their invincibility in the new era of change.

\subsection{Increase investment in research and development of blockchain technology to improve banks' own digital capabilities}

For traditional commercial banks, exploring digital development channels based on blockchain technology not only has high requirements on the operation and maintenance of internal systems of banks, but also requires commercial banks to have certain financial system development and operation capabilities. In the R\&D and maintenance stages, banks cannot do without high-tech talents with corresponding knowledge reserves and practical experience, which requires commercial banks to establish their own internal research teams and set up special technology and product R\&D departments to carry out product and system R\&D work [5]. At the early stage of digital construction of commercial banks, banks can cooperate with enterprises and universities to learn knowledge about digital development channels, follow the development trend of digital economy, increase the training of blockchain technology talents, pay attention to the application of blockchain technology in actual operation, and explore digital development channels suitable for themselves.

\subsection{Pay close attention to the forward-looking research of blockchain technology and actively participate in it}

Due to the rapid development of the digital economy, it has driven the rapid development of many financial technologies within the financial sector. Blockchain technology, as the underlying application technology of today's digital economy, requires commercial banks to explore their own digital development path by actively participating in international seminars and exchanges on related topics, paying close attention to the most cuttingedge development direction of blockchain technology, and at the same time strengthening cooperation and research on blockchain technology among financial industries. On top of drawing on a large number of domestic and international experiences in blockchain development, it should propose its own innovative research methods, establish its own blockchain ecosystem, and promote the rapid transformation and upgrading of banks.

\subsection{Establish a mechanism to protect the privacy of commercial banks' customers and keep the blockchain technology "safe"}

Since blockchain technology is still in the early stage of development, at the same time, commercial banks, as intermediaries of social and economic circulation, have a large amount of customer data information. How to identify which data is the private data of customers and which data is the analyzable data of customers has become a difficult problem for commercial banks to develop blockchain at present.

Based on this, commercial banks should establish a perfect customer privacy protection mechanism through a lot of practical research, combining domestic and foreign privacy protection paths and using cutting-edge ring signature, mixed coin and homomorphic plus technologies. By encrypting customers' data and allowing the bank's data system to open query channels only for those who have the right to use the key, this can greatly improve the security of passwords, effectively prevent malicious attacks from hackers and viruses, and further improve the customer privacy protection mechanism. At the same time, commercial banks should also establish a strong data security system to strictly prevent the leakage of customer information and establish a multi-layer prevention and control mechanism to ensure data security.

\section{Conclusion}

In the era of digital economy, the development of blockchain technology has created potential opportunities for the future development of commercial banks. However, the essence of digital transformation of commercial banks is still commercial banks. Blockchain finance plays a fundamental and innovative role in commercial banks. Through the use of qualitative analysis and literature research, this paper finds that the traditional development model has been unable to adapt to the current development of commercial banks, and the combination of commercial banks and blockchain technology has become an irreversible trend of the times. The emphasis of blockchain technology in the fourteenth five year plan indicates that the rapid development of blockchain finance will become an important opportunity for China's commercial banks to overtake in the development of international commercial banks. Relying on the blockchain, commercial banks integrate new industrial technologies such as big data and cloud computing, while building a complete digital ecosystem, deepen the in-depth development integration of blockchain and banking business, and actively promote the development of related financial derivatives, which will be the main trend of the development of commercial banks in the future.

\section{REFERENCES}

1. Li, Haiwei. Application of blockchain technology in mobile digital money order [D]. Nanjing University of Technology,2017. 
2. Lv Yongpeng, Yang Xinlian. Exploring the application of supply chain finance in China[J]. Business intelligence, 2013, 000(037):59-59.

3. Liu Zhijian, Xiao Yuxiu. The impact of Internet finance on commercial banks and its revelation[J]. Market Research, 2014(01):23-25.

4. Zhang Peng. Research on the impact of blockchain technology on traditional trade settlement methods of commercial banks [D]. University of International Business and Economics.

5. Li Tong. Research on transformation and innovation of profitability model of commercial banks in China in the process of supply-side reform:methods and paths[D]. Yunnan University of Finance and Economics, 2017. 